\title{
Prevalence and Perceptions about Migraine among Students and Patients in Khyber Pakhtunkhwa Province, Pakistan
}

\author{
Muhammad Zahid1 ${ }^{*}$, Aftab Alam Sthanadar',2, Muhammad Kaleem¹, Muhammad Latif1, \\ Iram Alam Sthanadar ${ }^{1}$, Pir Asmat Ali ${ }^{1}$, Irum Alam Sthanadar ${ }^{1}$, Muhammad Ismail ${ }^{1}$, \\ Noreen Imtiaz ${ }^{1}$, Mudassir Shah ${ }^{1,3}$ \\ ${ }^{1}$ Department of Zoology, Islamia College University, Peshawar, Pakistan \\ ${ }^{2}$ Department of Zoology and Animal Sciences, Post Graduate College Dargai, Malakand, Pakistan \\ ${ }^{3}$ Government Degree College Dara Adam Kheil, Kohat, Pakistan \\ Email: ${ }^{\text {mzahidsafi75@yahoo.com }}$
}

Received 15 March 2014; revised 16 April 2014; accepted 1 May 2014

Copyright (C) 2014 by authors and Scientific Research Publishing Inc.

This work is licensed under the Creative Commons Attribution International License (CC BY). http://creativecommons.org/licenses/by/4.0/

c) (7) Open Access

\section{Abstract}

Migraine is a common neurological disorder, prevalent in almost all over the world population and being considered as the $13^{\text {th }}$ major medical disorder among other major disorders across the globe, generally characterized with unilateral or bilateral headache, nausea and vomiting. In developing world, countries like Pakistan, most of the patients are not well aware of migraine headaches, as usually taken for simple headache due to no proper awareness. Usually it is not a contagious condition and most of the time it is recoded as episodic in nature. To assess the migraine, its frequency, symptoms, usual triggers and patients' perceptions, the present study was conducted. For this purpose a prospective study was carried out based on properly designed questionnaire. The data were collected from 7 educational institutes, including students of Islamia College Peshawar, University of Peshawar, Khyber Medical College, Engineering University Peshawar, Fatima Jinnah College Peshawar, Commerce College Peshawar and Agriculture University Peshawar. Part of data was also collected from patients visiting Khyber Teaching Hospital Peshawar for their headache problems. A total of 270 questionnaires were filled up. About $81(30.00 \%)$ headache cases were recorded, fulfilling the International criteria of Headache. Out of 81 recorded cases, 31 $(38.3 \%)$ patients were of migraine with aura and $50(61.7 \%)$ were of migraine without aura condition. The prevalence of migraine in the study population was found to be $30.00 \%$. The frequency of female migraine patients was higher i.e. $31(34.1 \%)$ than the male sufferers $50(27.9 \%)$. The present study clearly showed that the higher frequency $(65.0 \%)$ of migraine exists in the age group of above 30 years age. The most prevalent trigger was found to be tension, which was in pa-

\footnotetext{
*Corresponding author.

How to cite this paper: Zahid, M., Sthanadar, A.A., Kaleem, M., Latif, M., Sthanadar, I.A., Ali, P.A., Sthanadar, I.A., Ismail, M., Imtiaz, N. and Shah, M. (2014) Prevalence and Perceptions about Migraine among Students and Patients in Khyber Pakhtunkhwa Province, Pakistan. Advances in Bioscience and Biotechnology, 5, 508-516.
}

http://dx.doi.org/10.4236/abb.2014.56061 
rallel followed by the lack of sleep or oversleeping. A family history for migraine was found in migraine patients. Usually the condition was found to limit the routine activities of patients. The present study recorded that most of the migraine patients $(\mathbf{4 0 . 2 \% )}$ ) did not visit doctors at hospital and preferably go for self-medication, which really needs a public awareness in order to properly cope this neurological disorder, which may pose future medical complications as well.

\title{
Keywords
}

\author{
Migraine, Migraine with Aura (MWA), Migraine without Aura (MWOA), International Headache \\ Society (IHS)
}

\section{Introduction}

Migraine is a considerable health problem in developed as well as in underdeveloped countries [1] [2]. It is a long lasting neurological disorder generally with common symptoms of headaches and nausea. Previous studies have shown that the frequency of migraine is three times more in women than men [3]. Usually the headache caused by migraine is unilateral and throbbing in nature which commonly lasts for 2 to 72 hours [4]. Clinically, signs and symptoms of migraine are vomiting, nausea, weakness and sensitivity to light and sound [5]. The very detail account of migraine is still unknown. However the latest studies suggest that, it may be due to hyper excitability of the cerebral cortex or due to the abnormality of the neurons in trigeminal nucleus of brainstem [6]-[8].

Migraine is a common neurological disorder, prevalent up to $6 \%$ in men and $18 \%$ in females with the highest prevalence age from 25 to 55 years [9]. Presently, it has been medically considered as a high public health burden on the society, being a $19^{\text {th }}$ major health problem across the world population [7] [8]. It has been significantly affecting the day-to-day routine activities and leading to immediate and temporary disability concerns [9]. Regarding its different types, it is classified into six subtypes with two major and two usual types i.e. migraine without aura (MWOA) and migraine with aura (MWA) [2] [4] [7] [8].

Migraine with aura is also known as a classical type of migraine. In this type of migraine, the patient usually feels aura in half an hour before the actual pain starts. It has transient focal neurological symptoms including visual disturbance which is followed by headache [10], while migraine without aura is the next most common type of migraine. Its common symptoms include sudden onset of throbbing, unilateral pain in head, which becomes more severe on movement and may last for 4 - 72 hours if untreated [11].

Presently we aimed to assess the prevalence of these 2 major types of migraine-migraine with aura (MWA) and migraine without aura (MWOA) to record the students and patients perceptions about migraine across Peshawar city at Khyber Pakhtunkhwa Province, Pakistan. Some studies have been conducted before, but most of the people are unaware of this problem, and even qualified strata of the society like students is not well aware and takes it as a common, minute and neglected medical hazard.

\section{Methodology}

\subsection{Study Design at Hospital}

This was a descriptive study using a questionnaire as a data collecting tool. The questionnaire was developed in keeping with the objectives of the study in accordance with International headache society criteria [6] [7]. Open and closed ended questions were used. The questionnaire was pilot tested, and the investigators were properly consulted before the start of the study. Questionnaires were patiently filled by investigators in the patients' waiting room. Before filling, all patients in the waiting room were properly informed about the study. They were requested to participate in the study, based on their availability and convenience. Keeping in view the Pakhtun-cultural aspect of the province, male investigators collected data from male patients and female investigators were used to collect data from female patients. The study investigators administered the questionnaire, after the participants agreed to participate. The participating patient signed a consent form after assurance of confidentiality of their data provided to them. Questions were asked in local language. Most of the patients were belonging to Pathan Tribe and were Pashto speaking, a dominantly spoken language across the whole of Khyber Pakhtunkhwa province in Pakistan. The interviewers asked the questions and filled out the questionnaire accordingly. 
Explanations were provided for any questions requiring clarification.

\subsection{Sampling at University Campuses}

Similar questionnaires were administered at University Campuses. Snow ball sampling method was quite helpful at student level to collect data regarding migraine in university students. That is why, only students declared with headache were included and data were collected from such students at campuses level. However at hospital, only those patients were invited to participate in the studies, who were visiting physicians for headache problem.

\subsection{Study Population}

The study population comprised of students and patients visiting the Khyber Teaching Hospital (KTH) at Peshawar city in KP province in Pakistan. The study was conducted in January to December 2013. KTH is among the main hospitals across Khyber Pakhtunkhwa province. About one hundred patients are seen at KTH daily by working doctors, from Monday to Saturday. Pakistan is a developing country with the majority of the population not well educated and underprivileged. KTH is a government and teaching hospital, providing primary, secondary and tertiary level care across the province. Generally patients visiting KTH were of middle class with optimum economic status. While students visiting KTH were well educated and socioeconomically better off than the rest of the population. As majority of them were graduate level students. Some female students were not agree for taking part in the study, due to family pressure and Purdah-culture, meaning females should not actively participate in activities which males can do. They were simply excluded from the study, however about 9-refusal cases were faced at hospital which were either due to parents in hurry or were mothers with more children and their children with annoying and irritating behavior, like weeping and noise making.

\subsection{Study Inclusion-Exclusion Criteria}

Participants at hospital and at university campuses with diagnosed 02 major migraine types were included in the study sample, irrespective of their age, sex, location, profession and education. While patients and students with other types of migraine and multiple symptoms were not included in the study, for the sake of credible results and valid data. As here in the present study only individuals with MWA and MWOA were included, not other than these two major types of headache condition.

\subsection{Ethical Certificate}

Ethical certificates regarding the present study were obtained from Heads of the concerned Institutes in black and white.

\subsection{Data Analysis}

As we designed a descriptive study without applying any statistical tests on the data, sample size estimation was not taken as an issue. Data collecting-questionnaire was coded and the answers were grouped together. Computer software like Epi-info and Graph Pad Prism $5^{\text {th }}$ version were used for data entry and analysis.

\section{Results}

A total of 270 samples were collected from patients and students visiting Khyber Teaching Hospital Peshawar (KTH) from January to December 2013. Of 270 cases, 81 (30.00\%) patients were diagnosed as migraine patients and $189(70.00 \%)$ patients have non migraine headaches. Out of 81 patients, 31 (38.3\%) were diagnosed as migraine with aura and 50 (61.7\%) patients have migraine without aura. Migraine type of headaches were related with age at the time of presentation, intensity of the headache, marital status, family history, gender, routine activity limitations, age at the onset of first attack of migraine, associated characters, treatment modality, associated symptoms, quality of headache, visits to doctor for treatment and triggers of migraine headache were recorded in the study sample.

Table 1 shows age wise distribution of study sample. It was found that patients above 30 years age have higher prevalence (65.0\%) of migraine. Out of that, there were 10.0\% MWA and 55.0\% MWOA. While patients with age less than 30 years have less migraine attacks. As there were 44.4\% MWA and 14.8\% MWOA recorded 
Table 1. Migraine according age, sex, marital status, family history and intensity level.

\begin{tabular}{|c|c|c|c|c|}
\hline & NMH (\%) & MWA (\%) & MWOA (\%) & Total (\%) \\
\hline \multicolumn{5}{|l|}{ Age groups: (Years) } \\
\hline $16-20$ & $82(78.9)$ & $09(8.6)$ & $13(12.5)$ & $104(38.5)$ \\
\hline $21-29$ & $99(67.8)$ & $21(14.4)$ & $26(17.8)$ & $146(54.1)$ \\
\hline$>30$ & $07(35.0)$ & $02(10.0)$ & $11(55.0)$ & $20(7.4)$ \\
\hline \multicolumn{5}{|l|}{ Sex: } \\
\hline Female & $60(65.9)$ & $10(11.0)$ & $21(23.1)$ & 91 (33.7) \\
\hline Male & $129(72.1)$ & $21(11.7)$ & $29(16.2)$ & $179(66.3)$ \\
\hline \multicolumn{5}{|l|}{ Marital status: } \\
\hline Un-married & $17(34.0)$ & $16(32.0)$ & $17(34.0)$ & 50 (18.5) \\
\hline Married & $172(78.2)$ & $22(10.0)$ & $26(11.8)$ & $220(81.5)$ \\
\hline \multicolumn{5}{|l|}{ Family history: } \\
\hline First degree relative & 100 (69.9) & $23(16.1)$ & $20(14.0)$ & $143(53.0)$ \\
\hline Second degree relative & $43(65.2)$ & $04(6.1)$ & 19 (28.7) & $66(24.4)$ \\
\hline No family history & $46(75.4)$ & $04(6.6)$ & $11(18.0)$ & $61(22.6)$ \\
\hline \multicolumn{5}{|l|}{ Intensity of disease: } \\
\hline Mild & $32(61.5)$ & $08(15.4)$ & $12(23.1)$ & $52(19.3)$ \\
\hline Moderate & $114(74.5)$ & $15(9.8)$ & $24(15.7)$ & $153(56.6)$ \\
\hline Severe & $43(66.1)$ & $08(12.4)$ & $14(21.5)$ & $65(24.1)$ \\
\hline
\end{tabular}

*G.Total $(\%)=$ Grand total $(\%)=270(100.00)$.

in the age group from 21 - 29 years. Similarly, there were 8.6\% and 12.5\% MWA and MWOA in age group from 16 - 20 years. Regarding sex wise, migraine was found more in females than males. There were $34.1 \%$ headaches in females as compared to $27.9 \%$ headaches in males as shown in Table 1 . In relation to marital status, the occurrence of migraine was higher (66.0\%) in unmarried subjects (MWA 32.0\%, MWOA 34.0\%) than in married patients as recorded in Table 1 . For finding the relation between family history and migraine, three categories were formed as $1^{\text {st }}$ degree relatives, $2^{\text {nd }}$ degree relatives and no family history. The high prevalence (30.1\%) of migraine was found in $2^{\text {nd }}$ degree category. Where $16.1 \%$ headaches were MWA and $14.0 \%$ were MWOA, as seen in Table 1. Regarding intensity of headache, headaches were divided into three categories i.e. mild, moderate and severe. It was found that sever condition of headache were more common (38.5\%) than mild (25.5\%) and severe condition (33.9\%), as tabulated in Table 1.

Table 2 shows the age of very first attack in migraine patients. It was found that the most frequent age of migraine first attack was age above from 30 years. As there were $66.7 \%$ patients were suffering from migraine, including $66.0 \%$ MWA and $00.0 \%$ MWOA within the age group above 30 years age at the onset of $1^{\text {st }}$ attack. Table 3 shows the symptoms of migraine in the study population. Among migraine patients (MWA, MWOA) vomiting, nausea and photophobia was the most reported symptoms (80.0\%). Among associated characters with migraine headaches, Throbbing, expoding and aching were the most common and associated characters with migraine, as shown in Table 3. Data related to migraine relation to daily working efficiency was arranged in Table 4. Subjects about $50.0 \%$ were affected by migraine headache condition as compared to $27.4 \%$ non-affected subjects. Table 5 shows management of migraine condition. The most effective treatment modality for migraine headache was found to be body massage i.e. 50\% (16.4\% MWA, 23.6\% MWOA). Table 6 shows people perceptions about migraine headaches. It was found that only $24.8 \%$ Patients visits doctors for their treatment, while $40.2 \%$ patients do not visit doctors for their treatment. The distribution is clearly seen in Table 6 .

\section{Discussion}

Across low economic countries like Pakistan, majority of the people are unaware of the proper treatment for common headaches especially migraine. They generally do not visit doctors if they experience any headache and just go for the easily available medicines to treat their headaches. Commonly quakes are consulted and simply 
Table 2. Migraine first attack in different age groups.

\begin{tabular}{ccccc}
\hline & NMH (\%) & MWA (\%) & MWOA (\%) & Total (\%) \\
\hline Age at first attack: & & & & \\
$>15$ & $59(79.7)$ & $6(8.1)$ & $9(12.2)$ & $20(16.1)$ \\
$15-20$ & $89(71.8)$ & $15(12.1)$ & $08(34.8)$ & $124(46.0)$ \\
$21-25$ & $12(52.2)$ & $03(13.0)$ & $04(66.7)$ & $23(8.5)$ \\
$>26$ & $2(33.3)$ & $0(0.00)$ & $09(20.9)$ & $06(2.2)$ \\
Not known & $27(62.8)$ & $07(16.3)$ & & $43(15.9)$ \\
\hline
\end{tabular}

${ }^{*}$ G.Total $(\%)=$ Grand total $(\%)=270$ (100.00).

Table 3. Symptoms, associated characters and different triggers of migraine.

\begin{tabular}{|c|c|c|c|c|}
\hline & NMH (\%) & MWA (\%) & MWOA (\%) & Total (\%) \\
\hline \multicolumn{5}{|l|}{ Symptoms: } \\
\hline Weakness & $28(66.7)$ & $04(9.5)$ & $10(23.8)$ & $42(15.5)$ \\
\hline Nausea & $16(80.0)$ & $01(5.0)$ & $03(15.0)$ & $20(7.4)$ \\
\hline Vomiting & $13(76.5)$ & $01(5.9)$ & $03(17.6)$ & $17(6.3)$ \\
\hline Photophobia & $23(69.7)$ & $06(18.2)$ & $04(12.1)$ & $33(12.2)$ \\
\hline Phonophobia & $16(72.8)$ & $03(13.6)$ & 03 (13.6) & $22(8.2)$ \\
\hline Nausea + photophobia & $08(61.5)$ & $01(7.7)$ & $04(30.8)$ & $13(4.8)$ \\
\hline Nausea + phonophobia & $04(36.4)$ & $04(36.4)$ & $3(27.2)$ & $11(4.1)$ \\
\hline Vomiting + photophobia & $11(52.4)$ & $04(19.0)$ & $06(28.6)$ & $21(7.8)$ \\
\hline Vomiting + phonophobia & $07(77.8)$ & $01(11.1)$ & $01(11.1)$ & 09 (3.3) \\
\hline Weakness + photophobia & $33(80.5)$ & $05(12.2)$ & $03(7.3)$ & $41(15.2)$ \\
\hline Weakness + phonophobia & $13(65.0)$ & $04(20.0)$ & $03(15.0)$ & $20(7.5)$ \\
\hline Nausea + vomiting + photo & $02(20.0)$ & $02(20.0)$ & $06(60)$. & $10(3.7)$ \\
\hline Nausea + weakness & $01(33.3)$ & $02(66.7)$ & $03(1.1)$ & * \\
\hline Vomiting + weakness & $01(25.0)$ & $03(75.0)$ & $04(1.5)$ & $*$ \\
\hline Nausea + vomiting & $01(50.0)$ & $01(50.0)$ & $02(0.7)$ & \\
\hline Nausea + weakness + photo & $02(100.0)$ & $*$ & * & $02(0.7)$ \\
\hline \multicolumn{5}{|l|}{ Assocaited characters: } \\
\hline Throbbing & $07(58.3)$ & $02(25.0)$ & 03 (16.7) & $12(4.4)$ \\
\hline Exploding & $17(48.6)$ & $06(17.1)$ & $12(34.3)$ & 35 (13.0) \\
\hline Pressure like & 70 (72.9) & $11(11.5)$ & $15(15.6)$ & $96(35.6)$ \\
\hline Aching & $39(72.2)$ & 09 (16.7) & $06(11.1)$ & $54(20.0)$ \\
\hline Burning & $20(69.0)$ & $02(6.9)$ & $07(24.1)$ & 29 (10.7) \\
\hline Dullness like & $28(82.3)$ & $02(5.9)$ & $04(11.8)$ & 34 (12.6) \\
\hline Throbbing + pressure like & $03(75.0)$ & $01(25.0)$ & $*$ & $04(1.5)$ \\
\hline Throbbing + dullness & $04(66.7)$ & $02(33.3)$ & * & $06(2.2)$ \\
\hline \multicolumn{5}{|l|}{ Different triggers: } \\
\hline Tension & $102(71.3)$ & $18(12.6)$ & $23(16.1)$ & $143(52.9)$ \\
\hline Napping/oversleeping & $26(63.4)$ & $07(17.1)$ & 08 (19.5) & $41(15.2)$ \\
\hline Bright light & $13(59.1)$ & 03 (13.6) & $06(27.3)$ & $22(8.2)$ \\
\hline Menstruation & $15(68.2)$ & $02(9.1)$ & $05(22.7)$ & $22(8.2)$ \\
\hline Travel & $03(75.0)$ & $01(25.0)$ & $04(1.5)$ & * \\
\hline Skipping meal & $13(92.8)$ & ${ }^{*}$ & $01(7.2)$ & $14(5.2)$ \\
\hline Bending neck downward & 10 (76.9) & * & $03(23.1)$ & $13(4.8)$ \\
\hline Tension + bright light & $07(77.8)$ & * & $02(22.2)$ & 09 (3.3) \\
\hline Tension + menstruation & $*$ & $*$ & $02(100.0)$ & $02(0.7)$ \\
\hline
\end{tabular}

*G.Total(\%) = Grand total $(\%)=270(100.00)$ 
Table 4. Migraine in relation to routine activity.

\begin{tabular}{|c|c|c|c|c|}
\hline & NMH (\%) & MWA (\%) & MWOA (\%) & Total (\%) \\
\hline \multicolumn{5}{|l|}{ Routine activity: } \\
\hline No effect & $156(72.6)$ & $22(10.2)$ & $37(17.2)$ & 215 (79.6) \\
\hline Effect on work & $33(60.0)$ & 09 (16.4) & 13 (23.6) & $55(20.4)$ \\
\hline
\end{tabular}

${ }^{*}$ G.Total $(\%)=$ Grand total $(\%)=270(100.00)$.

Table 5. Treatment modalities of migraine.

\begin{tabular}{|c|c|c|c|c|}
\hline & NMH (\%) & MWA (\%) & MWOA (\%) & Total (\%) \\
\hline \multicolumn{5}{|c|}{ Treatment modality: } \\
\hline Sleep & $105(77.2)$ & $14(10.3)$ & 17 (12.5) & $136(50.4)$ \\
\hline Body massage & $08(50.0)$ & $02(12.5)$ & $06(37.5)$ & $16(5.9)$ \\
\hline Dark quiet room & 17 (56.7) & $06(20)$ & 07 (23.3) & 30 (11.1) \\
\hline Medications & $29(65.9)$ & 05 (11.4) & $10(22.7)$ & 44 (16.3) \\
\hline Eating & $11(78.6)$ & * & $03(21.4)$ & $14(5.2)$ \\
\hline Drinking coffee & 19 (63.3) & 04 (13.4) & 07 (23.3) & 30 (11.1) \\
\hline
\end{tabular}

${ }^{*}$ G.Total $(\%)=$ Grand total $(\%)=270$ (100.00).

Table 6. Patients' attitude regarding migraine and hospital visits.

\begin{tabular}{ccccc}
\hline & NMH (\%) & MWA (\%) & MWOA (\%) & Total (\%) \\
\hline Patients attitude: & & & & \\
Do not visit hospital & $55(59.8)$ & $14(15.2)$ & $23(25.0)$ & $92(34.1)$ \\
Visit hospital & $134(75.3)$ & $17(9.6)$ & $27(15.2)$ & $178(65.9)$ \\
\hline
\end{tabular}

${ }^{*}$ G.Total $(\%)=$ Grand total $(\%)=270$ (100.00).

tablets are acquired to kill their headache. Whenever the situation gets enough worse and the headache did not respond to self-medication even then a practicing physician is consulted. Patient goes to a neurologist only when the headache persists for a longer time, and limiting the routine activities of the patient. That is why the present study was designed to know the different characteristics of headache especially migraine type of headaches and to report patients response regarding migraine headaches.

Based on previous studies, migraine was recorded in patients who are in the productive stage of their age. Likely, it is evident from the present study that a high rate of migraine headaches were recorded in people at the age above 30 years age, as in Table 1. Previous study of Murtaza et al. [12] has reported a high frequency of migraine in the age range of 21 - 30 years. Similarly, Queiroz et al. [13] also recorded a high prevalence of migraine headache in people with age of 30 - 39 years. Likely, the work of Al-Shimmery [14] supported a high frequency of migraine in the age range of 30 - 39 years. Similarly, Boru et al. [15] reported a high prevalence of migraine in people with age of 35 - 45 years. Their findings are quite in line with our work. Boru et al., [15] work suggests that the frequency of Margarine regarding age is varying. And the situation can be enough different across different countries at globe. This is quite debatable and needs further findings to clearly depict the situation.

The incidence of headache was found $30.00 \%$ in the present study. Studies like assessing migraine are quite scarcely reported across our country. Previously Bokhari et al. [5] worked on migraine in our adjacent province, at Lahore in Punjab. Bokhari et al. [5] reported 34.8\% incidence rate of migraine in college going girls of Lahore. Our findings are in line with his findings. A slight change in frequency is recorded in our study sample across province of Khyber Pakhtunkhwa. In Asian countries, like in Turkey, Celik et al. [16] reported be 19.9\% prevalence of migraine in Turkey. Sanchez et al. [17] has reported 29.4\% prevalence rate of migraine in Romanian population in Europe. Bigal et al. [18] recorded the almost similar prevalence rate i.e. $30.4 \%$ among hos- 
pital employees. Similarly Queiroz [13] found the one year prevalence rate of migraine to be $15.2 \%$ in Brazil in 2009. All previous studies support a different rate of migraine prevalence across different countries. Which may be associated with different factors? This really needs further findings why the rate is variable? That may include variation in geography, temperature, age of population and working status of individuals etc.

Regarding sex, frequency of migraine is more in females as compared to males, as we see in Table 1. In our present study sample, the frequency of MWA was $11.0 \%$ and $23.1 \%$ were MWOA in females population. Likely, Hamirani et al. [19] reported about 63.8\% migraine in females and 36.2\% in males. Our study findings are well supported by Hamirani et al. [19] and the work of Sanvito et al. [20]. Sanvito et al. [20] reported 54.4\% migraine in females and 28.3\% migraine in female population in their study sample. Domingues et al. [21] reported $62.2 \%$ of females and $37.8 \%$ of males suffered from migraine headaches. Jensen and Stovner, [22] also reported a high prevalence in female population.

The present study recorded a frequency of 30\% migraine headaches in local population of Peshawar and $70 \%$ of non-migraine headaches across the study population. Majority of the patients included in the study were belonging to the middle class of the society and others including students were engaged in academic activities. It was concluded that patients of migraine without aura (MWOA) were more (61.7\%) than the migraine with aura (MWA) patients (38.3\%), as seen in Table 1. Our present findings recorded that migraine is common in unmarried sufferers than the married patients. These results are consistent with the previous results [13]. This may be due to the stress of the education and social issues of females across our country.

Based on previous studies, it was found that migraine runs in families. Our study showed a positive family history of migraine. We recorded that $34.8 \%$ of the patients have family history of migraine than non-migraine headaches. The argument positively supports the relation between migraine and family history. Our work is consistent with the study of Bokhari et al. [5]. Their work reported 50\% positive family history in migraine patients. Similar findings were reported by Al-Shimmery [14]. Recently Al-Shimmery [14] found 61\% magraine patients were having positive family history of migraine. More over findings has recorded 70\% of positive family history [23]. Our work is strongly supported by previous studies. Regarding intensity of migraine, presently we recorded three types of headaches. Out of which mild type of headache condition was high (38.5\%) as compared to severe and moderate conditions of headache, as seen in Table 1.

Regarding age of headache first attack, our data concluded that headache is more frequent in age group above 30 years age. The situation may vary in different countries due to different socio-economic conditions and difference in standard of living. Age related distribution of headache is listed in Table 2. Table 3 listed different symptoms of headaches. Vomiting, nausea and photophobia were recorded as the most common symptoms of migraine in the study population. However this generalization may not be applicable everywhere due to sample size. Among associated characters with headache, "throbbing, exploding and aching" characters were more frequent character associated with headaches in our study. The situation may vary in case of other studies. The presence of triggers is quite common in migraine patients. The different types of migraine may be due to the same trigger. Here we recorded that tension and menstruation were most common triggers in patients among other triggers like napping, oversleeping, bright light and menstruation in our study population, as in Table 3. Our results are well supported by the study of Kelman [24]. The work of Kelman [24] recoded stress or tension as a common trigger for migraine. Rothrock et al. [25] also reported stress or tension to be more common trigger in his study. In their study population 59\% study sample was recorded with tension as the common trigger for migraine attack. More over the work of Neut et al. [26] showed stress or tension and lack of sleep as most prevalent triggers for migraine. Contrarily, findings of Al-Shimmery [14] are different. Who reported stress and increasing physical activity to be the two most prevalent trigger for migraine in Iraqi Kurdish patients. The difference may be because of variation in geography.

It was also known that migraine limit the routine activity more than the non-migraine headache sufferers. As long as the headache characteristics are concerned, different treatment modalities are used for migraine condition, including sleeping, napping, dark room stay, avoiding light and massage. In our findings massage was the most effective treatment modality in migraine patients, as seen in Table 4, Table 5. Regarding people perception about headache, our study population was not well aware of migraine. Most of the subjects took it as usual headache case and never seriously considered it as neurological disorder to consult physician for proper treatment in order to avoid any future complications. In our study we see (Table 6) about $42.2 \%$ migraine patients were not visiting doctor and hospitals, which really need a proper awareness in order to avoid any possible future neurological complication in the long run. 


\section{Conclusion}

The conclusion of this study is that the incidence of migraine in Peshawar was found to be $30.0 \%$, while $70 \%$ of the headaches were non-migraine headaches. In this study, the percentage of migraine was found higher in females than that in males. The present study clearly recorded that the occurrence of the present medical disorder was significantly higher in the age group above 30 years of age. Among the under lying causes, tension was found which was followed by menstruation condition. In this study a positive family history was noted among the patients of migraine. A higher percentage of migraine was observed in unmarried patients than that in married sufferers. Massage was the most efficient treatment modality identified in the present study which was primarily recorded in all migraine patients. Most of the migraine patients did not visit doctor for their treatment and take over the counter medicines. Vomiting, nausea and photophobia were common symptoms of migraine. Characters like throbbing, exploding and aching were the most associated characters attached with migraine headache in the study population. Migraine was observed to limit the routine activities of the patients and also disturbed their family life.

\section{Acknowledgements}

We are thankful to Professor Philippe Billiald for the critical reading of manuscript. Many thanks to the Physicians and Doctors for extending all the possible help.

\section{References}

[1] Natoli, J.L., Manack, A. and Dean, B. (2010) Global Prevalence of Chronic Migraine: A Systematic Review. Cephalalgia, 30, 599-609.

[2] Wang, S.J. (2003) Epidemiology of Migraine and Other Types of Headache in Asia. Current Neurology and Neuroscience Reports, 3, 104-108. http://dx.doi.org/10.1007/s11910-003-0060-7

[3] Stovner, L.J., Zwart, J.A., Hagen, K., Terwindt, G.M. and Pascual, J. (2006) Epidemiology of Headache in Europe. European Journal of Neurology, 13, 333-345. http://dx.doi.org/10.1111/j.1468-1331.2006.01184.x

[4] Mathew, N.T., Stubits, E. and Nigam, M.P. (1982) Transformation of Episodic Migraine into Daily Headache: Analysis of Factors. Headache, 22, 66-68. http://dx.doi.org/10.1111/j.1526-4610.1982.hed2202066.x

[5] Bokhari, F.A., Sami, W., Shakoori, T.A., Ali, S.A. and Qureshi, G.A. (2008) Clinical Characteristics of 226 College-Going Female Migraineurs in Lahore, Pakistan-Putting ICHD-2 to the Road Test. Neuroendocrinology Letters, 229, 965-970.

[6] Dodick, D. and Gargus, J.J. (2008) Why Migraines Strike. Scientific American, Inc., USA, 56-63.

[7] Headache Classification Committee of the International Headache Society (1988) Classification and Diagnostic Criteria for Headache Disorders, Cranial Neuralgias and Facial Pain. Cephalalgia, 8, 1-96.

[8] Headache Classification Subcommittee of the International Headache Society (2004) The International Classification of Headache Disorders: 2nd Edition. Cephalalgia, 24, 9-160.

[9] Lipton, R.B., Stewart, W.F. and Korff, M.V. (1997) Increasing the Options for Effective Migrane Management: Articles Burden of Migraine Societal Costs and Therapeutic Opportunities. Neurology, 48, S4-S9. http://dx.doi.org/10.1212/WNL.48.3_Suppl_3.4S

[10] Westermann, C.J., et al. (2003) The Prevalence and Manifestations of Hereditary Hemorrhagic Telangiectasia in the Afro-Caribbean Population of the Netherlands Antilles: A Family Screening. American Journal of Medical Genetics Part A, 116, 324-328. http://dx.doi.org/10.1002/ajmg.a.10002

[11] Klein, E. and Spencer, D.C. (2009) Migraine Frequency and Risk of Cardiovascular Disease in Women. Neurology, 73, 42- 43. http://dx.doi.org/10.1212/WNL.0b013e3181b7c1d8

[12] Murtaza, M., Kisat, M., Daniel, H. and Sonawalla, A.B. (2009) Classification and Clinical Features of Headache Disorders in Pakistan: A Retrospective Review of Clinical Data. PloS One, 4, e5827. http://dx.doi.org/10.1371/journal.pone.0005827

[13] Queiroz, L.P., Peres, M.F.P., Piovesan, E.J., Kowacs, F., Ciciarelli, M.C., Souza, J.A. and Zukerman, E. (2009) A Nationwide Population-Based Study of Migraine in Brazil. Cephalalgi, 29, 642-649. http://dx.doi.org/10.1111/j.1468-2982.2008.01782.x

[14] Al-Shimmery, K.E. (2010) Precipitating and Relieving Factors of Migraine Headache in 200 Iraqi Kurdish Patients. Oman Medical Journal, 25, 212-217. http://dx.doi.org/10.5001/omj.2010.59

[15] Boru, U.T., Kocer, A., Luleci, A., Sur, H., Tutkan, H. and Atli, H. (2005) Prevalence and Characteristics of Migraine in 
Women of Reproductive Age in Istanbul Turkey: A Population Based Survey. Tohoku Journal of Experimental Medicine, 206, 51-59. http://dx.doi.org/10.1620/tjem.206.51

[16] Çelik, Y., Ekuklu, G., Tokuç, B. and Utku, U. (2005) Migraine Prevalence and Some Related Factors in Turkey. Headache: The Journal of Head and Face Pain, 45, 32-36. http://dx.doi.org/10.1111/j.1526-4610.2005.05007.x

[17] Sánchez, S.J., Peñas, C.F., García, R.J., Barrera, V.H., Blanco, C.A., Ceña, D.P. and Garrido, P.C. (2013) Prevalence of Migraine Headaches in the Romany Population in Spain: Sociodemographic Factors, Lifestyle and Co-Morbidity. Journal of Transcultural Nursing, 24, 6-13. http://dx.doi.org/10.1177/1043659612452008

[18] Bigal, M.E., Bigal, J.O., Bordini, C.A. and Speciali, J.G. (2001) Prevalence and Costs of Headaches for the Public Health System in a Town in the Interior of the State of São Paulo. Arquivos de Neuro-Psiquiatria, 59, 504-511. http://dx.doi.org/10.1590/S0004-282X2001000400005

[19] Hamirani, M.M., Ahmed, S. and Luhano, M.L. (2008) Frequency of Anxiety and Depression in Migraine-A Study of 102 Patients. JLUMHS, 194-198.

[20] Sanvito, W.L., Monzillo, P.H. and Peres, M.F. (1996) The Epidemiology of Migraine in Medical Students. Headache, 36, 316-319. http://dx.doi.org/10.1046/j.1526-4610.1996.3605316.x

[21] Domingues, R.B., Camila, C.H., Aquino, J.G., Santos, D.A Silva, A.P. and Kuster, G.W. (2006) Prevalence and Impact of Headache and Migraine among Pomeranians in Espiritosanto, Brazil. Arquivos de Neuro-Psiquiatria, 64, 954-957.

[22] Jensen, R. and Stovner, L.J. (2008) Epidemiology and Comorbidity of Headache. The Lancet Neurology, 7, $354-361$. http://dx.doi.org/10.1016/S1474-4422(08)70062-0

[23] Faiqa, Q. and Donald, L. (2003) Managing Headache in the Pediatric Emergency Department. Clinical Pediatric Emergency, 4, 159-170.

[24] Kelman, L. (2007) The Triggers or Precipitants of the Acute Migraine Attack. Cephalalgia, 27, 394-402. http://dx.doi.org/10.1111/j.1468-2982.2007.01303.x

[25] Rothrock, J., Rothrock, A.D. and Weibelt, S. (2009) Menstrually Related Migraine Manifesting as Status Migrainosus. Neurology, 72, A474.

[26] Neut, D., Fily, A., Cuvellier, J.C. and Vallee, L. (2012) The Prevalence of Triggers in Paediatric Migraine: A Questionnaire Study in 102 Children and Adolescents. The Journal of Headache and Pain, 13, 61-65. http://dx.doi.org/10.1007/s10194-011-0397-2 